\title{
Investigating the Rhythm of Attention on a Fine-Grained Scale: Evidence from Reaction Times
}

\author{
Benedikt Zoefel ${ }^{1,2}$ and Rodika Sokoliuk ${ }^{1,2}$ \\ ${ }^{1}$ Université Paul Sabatier, 31062 Toulouse Cedex 9, France and ${ }^{2}$ Centre de Recherche Cerveau et Cognition (CerCo), CNRS, UMR5549, Pavillon Baudot CHU \\ Purpan, 31052 Toulouse Cedex, France \\ Review of Song et al.
}

There is increasing evidence that the human brain does not continuously sample the environment; instead, perception is inherently rhythmic, alternating between phases of high and low receptiveness for stimulus input (Schroeder and Lakatos, 2009). This periodic modulation of neural processing might be seen as a rhythmic opening and closing of "windows of opportunity" for a stimulus to be perceived or processed (Buzsáki and Draguhn, 2004). Since perception and attention are tightly linked phenomena, these findings naturally raise the question whether attention is an inherently cyclic process as well. In addition to the fact that most evidence suggests that the periodic nature of perception relies on attentional processes (Schroeder and Lakatos, 2009), Landau and Fries (2012) directly demonstrated that attention indeed entails a rhythmic process. In their study, attention was cued to one of two possible positions and subjects had to detect a visual target that was presented at either of the two locations. Interestingly, detection probability was found to oscillate (at $4 \mathrm{~Hz}$ ) at both locations, but, critically, in an antiphasic fash-

Received May 26, 2014; revised Aug. 6, 2014; accepted Aug. 9, 2014.

This study was supported by a Studienstiftung des deutschen Volkes (German National Academic Foundation) scholarship to B.Z.

The authors declare no competing financial interests.

Correspondence should be addressed to Benedikt Zoefel, Centre de Re-

cherche Cerveau et Cognition (CerCo), Pavillon Baudot CHU Purpan, BP 25202, 31052 Toulouse Cedex, France. E-mail: zoefel@cerco.ups-tlse.fr.

DOI:10.1523/JNEUROSCI.2134-14.2014

Copyright $\odot 2014$ the authors $\quad 0270-6474 / 14 / 3412619-03 \$ 15.00 / 0$ ion: when the "window of opportunity" was open for one location, it was closed for the other, and vice versa, indicating that attention shifted at a frequency of 8 $\mathrm{Hz}$ (4 Hz per location).

In a recent publication in The Journal of Neuroscience, Song et al. (2014) used a paradigm similar to the one described in Landau and Fries (2012) and provided further evidence for a rhythmic component in visual attention. Again, subjects were presented with a cue that drew attention to one of two possible locations, then a visual target presented at one of the two locations had to be detected as fast as possible. Critically, the time between cue and target onset (SOA) was variable-thus, under the hypotheses of (1) rhythmic attentional sampling and (2) a reset of this sampling by the presented cue, the difficulty of target detection should not be constant with respect to time, but depend on-or covary with-the respective SOA.

The study of Song et al. (2014) is of particular interest, because it differs from the study by Landau and Fries (2012) in two points. First, instead of using the probability of target detection to investigate attention as a function of time, the relation between SOA and reaction time (RT) was examined here. Second, and most importantly, the authors present an elegant approach to characterize the rhythmicity of attention: by using timefrequency analyses, the authors were able to detect much finer changes in os- cillatory variables than when using conventional analyses in the frequency domain [such as fast Fourier transformation (FFT)] that assume stationary oscillatory signals. Thus, Song et al. (2014) might have been able to detect properties of attentional sampling that remained hidden in earlier studies.

Indeed, not only did the authors corroborate the rhythmic sampling of attention, originally demonstrated by Landau and Fries (2012), they also reported an additional effect that directly depended on the phase of the sampling rhythm: the RT distribution showed an oscillatory pattern (again, around $4 \mathrm{~Hz}$ ) that was in antiphase at cued and uncued locations and, strikingly, the power of the RT distribution in a broad frequency band (around 5-25 Hz) was phase-locked to this rhythm (i.e., RT power was highest at a certain phase and lowest at the opposite phase). The authors interpret their findings as an attentional sampling in the theta-band $(3-5 \mathrm{~Hz})$ that is coupled to changes in alpha-power (5-25 Hz), in line with phase-amplitude coupling of neural oscillations that is commonly found in electrophysiological recordings (Tort et al., 2010).

Although we share the excitement with which Song et al. (2014) present their work, we would like to raise two important points. First, the rhythmic fluctuations of RT were found for both cued and uncued locations, but in an antiphasic fashion. Thus, when a given SOA was favorable for detecting a target at one loca- 
tion (reflected by a relatively low RT), it was disadvantageous for the other. Consequently, and similar to the conclusion drawn by Landau and Fries (2012), an attentional sampling of $4 \mathrm{~Hz}$ at one of the two locations would indicate an overall sampling rhythm at a frequency of $8 \mathrm{~Hz}$. This finding is in accordance with an increasing amount of evidence for periodicity in perception that is tightly linked to brain rhythms in this frequency range for the visual system (Thut et al., 2012; Romei et al., 2012). Second, the power effect described by Song et al. (2014) covers a wide frequency range $(5-25 \mathrm{~Hz})$. Since, by definition, an oscillation is restricted to a narrow frequency range (Luck, 2005), we consider it problematic to assume an oscillatory component to underlie this finding, in particular because only one behavioral variable is investigated and conclusions about neural oscillations are indirect.

We propose a different scenario that could explain the data shown in Song et al. (2014). Assuming a "true" underlying attentional sampling at $4 \mathrm{~Hz}$ per location, it might be possible that the instantaneous state of this sampling affects the variability of responses (reflected in RT) in a cyclic manner. For instance, variability might be low when participants were attentive (i.e., at a phase of high receptiveness for visual input) and focused on the task, resulting in systematic responses with a low amount of RT fluctuations across trials. Conversely, variability might be high when participants were inattentive (i.e., at a phase of low receptiveness for visual input), resulting in unsystematic responses and a high amount of RT fluctuations across trials.

We illustrate our speculation with a (admittedly simplistic) simulation: four random signals (corresponding to the number of trials for all but one SOA in Song et al., 2014) were averaged before being transformed into the time-frequency domain. Critically, the signals were constructed such that their variance covaried with the phase of a hypothetical underlying $4 \mathrm{~Hz}$ wave (Fig. 1A) and thus, by construction, alternated between states of high and low variance. Two sets (i.e., $2 \times 4$ ) of signals were constructed, with the underlying $4 \mathrm{~Hz}$ wave in antiphase between the two sets, reflecting the two conditions ("valid" and "invalid") in Song et al. (2014). The average RT time courses (across "subjects"; see below) for both "conditions" (corresponding to Fig. $1 \mathrm{~B}$, top, in Song et al., 2014) are displayed in Figure $1 B$. Other properties of the simu-
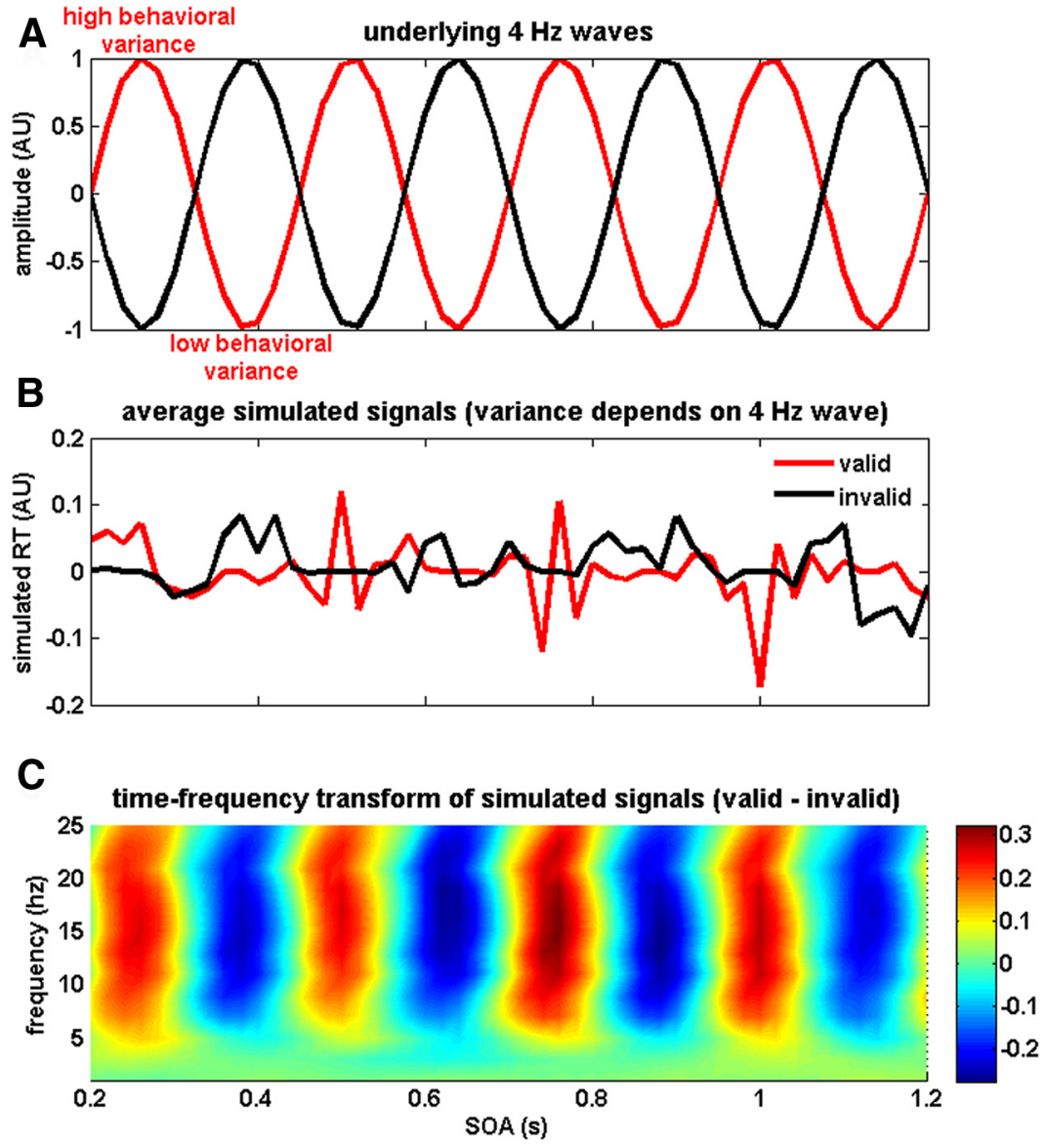

Figure 1. Rhythmic power changes can be explained by regular fluctuations in variance of a signal. $A$, Simulated signals $(2 \times$ 4 ; "valid" and "invalid" condition in red and black, respectively) were constructed whose variance depends on the phase of an underlying $4 \mathrm{~Hz}$ oscillation (highest variance at the peak of the $4 \mathrm{~Hz}$ oscillation, lowest variance at the trough). Note that the $4 \mathrm{~Hz}$ oscillations underlying the two conditions are in antiphase and are assumed to be reset at time $0 . \boldsymbol{B}$, This reset results in states of high and low variance that are consistent across signals and therefore do not cancel each other out after averaging the four signals. However, these states are in opposition between the two conditions (when variance is high in one condition, it is low in the other, and vice versa). C, Due to those rhythmic changes in variance, both the individual power profile of the two averaged signals (data not shown) and the average spectrotemporal profile of their difference show rhythmic changes in power (difference; colorcoded). Note the similarity of $\boldsymbol{B}$ and the raw RT shown in Song et al. (2014; their Fig. $1 B$, top), and the similarity of $\boldsymbol{C}$ and the spectrotemporal profiles shown in Song et al. (2014; Fig. 2A).

lated signals (e.g., the average) did not differ between states of high and low variance and the detailed parameters of the time-frequency analysis were as described in Song et al. (2014) (continuous complex Gaussian wavelet of order 4 for frequencies between 1 and $25 \mathrm{~Hz}$ in steps of $2 \mathrm{~Hz}$, sampling rate $50 \mathrm{~Hz}$ ). This procedure was repeated 49 times (corresponding to the number of subjects in Song et al., 2014) and the time-frequency transforms were averaged across "subjects," separately for each "condition." This analysis yields the RT power profiles corresponding to Figure $2 B$ in Song et al. (2014). The result of our simulation is shown in Figure 1C, representing the difference of the simulated power profiles between the two conditions (corresponding to Fig. $2 \mathrm{~A}$ in Song et al., 2014). Strikingly, this power difference exhibits regular fluctuations between positive and negative values at many frequencies, similar to the spectrotemporal profile shown by Song and colleagues (2014, their Fig. $2 A)$. Thus, rhythmic changes in a broad frequency range of a given spectrotemporal profile can theoretically be explained by regular fluctuations in the variability of the underlying signals.

In short, we believe that the study by Song et al. (2014) nicely underlines already reported evidence for perception and attention as cyclic processes, in line with electrophysiological results (Thut et al., 2012; Romei et al., 2012). However, the frequency range of the described alpha-power effect is too broad to reflect an oscillatory component and might reflect mere changes in RT variability that depend on the instantaneous state of in- 
put gating (i.e., high and low behavioral variability when attention is low and high, respectively).

\section{References}

Buzsáki G, Draguhn A (2004) Neuronal oscillations in cortical networks. Science 304:19261929. CrossRef Medline

Landau AN, Fries P (2012) Attention samples stimuli rhythmically. Curr Biol 22:1000-1004. CrossRef Medline
Luck SJ (2005) An introduction to the eventrelated potential technique. Cambridge: MIT.

Romei V, Gross J, Thut G (2012) Sounds reset rhythms of visual cortex and corresponding human visual perception. Curr Biol 22:807813. CrossRef Medline

Schroeder CE, Lakatos P (2009) Low-frequency neuronal oscillations as instruments of sensory selection. Trends Neurosci 32:9-18. CrossRef Medline

Song K, Meng M, Chen L, Zhou K, Luo H
(2014) Behavioral oscillations in attention: rhythmic alpha pulses mediated through theta band. J Neurosci 34:4837-4844. CrossRef Medline

Thut G, Miniussi C, Gross J (2012) The functional importance of rhythmic activity in the brain. Curr Biol 22:R658-R663. CrossRef Medline

Tort AB, Komorowski R, Eichenbaum H, Kopell N (2010) Measuring phase-amplitude coupling between neuronal oscillations of different frequencies. J Neurophysiol 104:1195-1210. CrossRef Medline 